\title{
ASYMPTOTIC BEHAVIOR OF SOLUTIONS TO THE KLEIN-GORDON EQUATION WITH A NONLINEAR DISSIPATIVE TERM
}

Dedicated to Professor Mutsuhide Matsumura on his sixtieth birthday

\author{
By
}

Takahiro MotaI

\begin{abstract}
We study the asymptotic behavior of the Klein-Gordon equation with a nonlinear dissipative term $\left|\partial_{t} w(t)\right|^{p-1} \partial_{t} w(t)(p>1)$ in $x \in R^{n}(n \geqq 1)$ and $t \geqq 0$. We prove that the energy of solutions does not converge to 0 as $t \rightarrow \infty$ for $p>1+2 / n$ if Cauchy data are suffciently small. We also prove that solutions of the above equation converge to suitable solutions of the linear Klein-Gordon equation in the energy space as $t \rightarrow \infty$ for $p>1+4 / n$ if $1 \leqq n \leqq 6$ and $1+4 / n<p<n /(n-6)$ if $n \geqq 7$.
\end{abstract}

Key Words. Klein-Gordon equation, nonlinear dissipative term, asymptotic behavior.

\section{Introduction and Results}

We consider the Cauchy problem for the nonlinear Klein-Gordon equation;

$$
\left\{\begin{array}{l}
\partial_{t}^{2} w(t)-\Delta w(t)+w(t)+f\left(\partial_{t} w(t)\right)=0 \\
w(0)=\phi, \quad \partial_{t} w(0)=\phi,
\end{array}\right.
$$

where $x \in \mathbb{R}^{n}, t \in \mathbb{R}^{+}=[0, \infty), f(u)=|u|^{p-1} u$ and $\Delta$ is the $n$-dimensional Laplacian. The asymptotic behavior of solutions of (1.1) was considered in Nakao [9]. He showed that

$$
\|W(t)\|_{e} \leqq C\|\Phi\|_{e}(1+t)^{-(2-n(p-1)) /(p-1)}
$$

for $1<p<1+2 / n$ and

$$
\|W(t)\|_{e} \leqq C\|\Phi\|_{e}(\log (1+t))^{-2 /(p-1)}
$$

for $p=1+2 / n$. Here $W(t)=\left(\begin{array}{c}w(t) \\ \partial_{t} w(t)\end{array}\right), \Phi=\left(\begin{array}{c}\phi \\ \psi\end{array}\right)$ and $\|\cdot\|_{e}$ is the energy norm

Received December 13, 1989. Revised June 18, 1990. 
defined by

$$
\|W(t)\|_{e}^{2}=\frac{1}{2}\left\{\|H w(t)\|_{2}^{2}+\left\|\partial_{t} w(t)\right\|_{2}^{2}\right\}
$$

where $\|\cdot\|_{2}$ is $L_{2}\left(\boldsymbol{R}^{n}\right)$-norm and $H$ is the positive selfadjoint operator $\sqrt{-\Delta+1}$ in $L_{2}$. Our aim of this paper is to investigate how the energy of solutions of (1.1) behaves as $t \rightarrow \infty$ in the case $p>1+2 / n$.

In order to state our results, we give the main notations used in this paper. We denote by $\|\cdot\|_{q}$ the norm in $L_{q}=L_{q}\left(\boldsymbol{R}^{n}\right)$. Let $H_{q}^{s}=H_{q}^{s}\left(\boldsymbol{R}^{n}\right)$ with $s \in \boldsymbol{R}$ and $1 \leqq q<\infty$ be the Sobolev spaces which are the completion of $C_{0}^{\infty}\left(\mathbb{R}^{n}\right)$ with norms

$$
\left.\|u\|_{s, q}=\| \mathscr{I}^{-1}\left(1+|\xi|^{2}\right)^{s / 2} \hat{u}(\xi)\right) \|_{q} .
$$

Here ${ }^{\wedge}$ denotes the Fourier transformation and $\mathscr{T}^{-1}$ is its inverse. Especially we denote by $H^{s}$ the usual Sobolev spaces. We note that $H^{s}=H_{2}^{s}$. For any interval $I \subset R$ and any Banach space $B$, we denote by $C^{k}(I ; B)$ the space of $B$ valued $C^{k}$-functions over $I$, and by $C_{w}(I ; B)$ the space of weakly continuous functions from $I$ to $B$, and by $C_{L}(I ; B)$ the space of functions from $I$ to $B$ that are strongly Lipschitz continuous. For any $q, 1 \leqq q \leqq \infty$, we denote by $L_{q}(I ; B)$ the space of $B$-valued $L_{q}$-functions on $I$.

We define an inner product in the energy space $H^{1} \times L_{2}$ by

$$
\left\langle\left(\begin{array}{l}
u_{1} \\
u_{2}
\end{array}\right),\left(\begin{array}{l}
v_{1} \\
v_{2}
\end{array}\right)\right\rangle_{e}=\frac{1}{2}\left\{\left\langle H u_{1}, H v_{1}\right\rangle+\left\langle u_{2}, v_{2}\right\rangle\right\},
$$

where $\langle$,$\rangle is L_{2}\left(\mathbb{R}^{n}\right)$-inner product. We note that $\|W(t)\|_{e}^{2}=\langle W(t), W(t)\rangle_{e}$.

We shall use the operator $\zeta(H)$ for suitable functions $\zeta(\cdot)$ as follows:

$$
\zeta(H) u=\mathscr{F}^{-1}\langle\zeta(\langle\xi\rangle) \hat{u}(\xi)) \quad \text { in } \mathcal{S}^{\prime},
$$

where $\langle\xi\rangle=\left(1+|\xi|^{2}\right)^{1 / 2}$ and $\mathcal{S}^{\prime}$ means the tempered distribution. We denote by $\{U(t)\}(t \in R)$ an unitary group in $H^{1} \times L_{2}$ defined by

$$
U(t)=\left(\begin{array}{cc}
\cos \{H t\} & H^{-1} \sin \{H t\} \\
-H \sin \{H t\} & \cos \{H t\}
\end{array}\right) .
$$

First we state a result of existence and uniqueness.

TheOREM 1. Let $n \geqq 1$ and $p>1$. Assume that $\Phi=\left(\begin{array}{c}\phi \\ \psi\end{array}\right) \in H^{2} \times H^{1} \cap L_{2 p}$. Then there exists a unique solution $w(t)$ of (1.1) which satisfies the following:

$$
\begin{gathered}
w(t) \in L_{\infty}\left(\mathbb{R}^{+} ; H^{2}\right) \cap C\left(\mathbb{R}^{+} ; H^{2}\right) \cap C^{1}\left(\mathbb{R}^{+} ; H^{1}\right) \cap C^{2}\left(\mathbb{R}^{+} ; L_{2}\right), \\
\partial_{t} w(t) \in L_{\infty}\left(\mathbb{R}^{+} ; H^{1}\right) \cap L_{p+1}\left(\boldsymbol{R}^{+} \times \boldsymbol{R}^{n}\right) \cap L_{\infty}\left(\mathbb{R}^{+} ; L_{2 p}\right), \\
\partial_{t}^{2} w(t) \in L_{\infty}\left(\mathbb{R}^{+} ; L_{2}\right) .
\end{gathered}
$$


And the following energy equality and inequalities hold:

$$
\begin{aligned}
& \|W(t)\|_{e}^{2}+\int_{0}^{t}\left\|\partial_{t} w(\tau)\right\|_{p+1}^{p+1} d \tau=\|\Phi\|_{e}^{2}, \\
& \|H W(t)\|_{e}^{2}+\int_{0}^{t}\left\langle\left|\partial_{t} w(\tau)\right|^{p-1}, p\left|\nabla \partial_{t} w(\tau)\right|^{2}+\left|\partial_{t} w(\tau)\right|^{2}\right\rangle d \tau \leqq\|H \Phi\|_{e}^{2}, \\
& \left\|\partial_{t} W(t)\right\|_{e}^{2}+p \int_{0}^{t}\left\langle\left|\partial_{t} w(\tau)\right|^{p-1},\left|\partial_{t}^{2} w(\tau)\right|^{2}\right\rangle d \tau \leqq\left\|\left(\begin{array}{c}
\phi \\
-H^{2} \phi-f(\phi)
\end{array}\right)\right\|_{e}^{2} \\
& \text { for } t \in R^{+} \text {, where } H W(t)=\left(\begin{array}{c}
H w(t) \\
H \partial_{t} w(t)
\end{array}\right), \partial_{t} W(t)=\left(\begin{array}{c}
\partial_{t} w(t) \\
\partial_{t}^{2} w(t)
\end{array}\right) \text { and } H \Phi=\left(\begin{array}{c}
H \phi \\
H \phi
\end{array}\right) \text {. }
\end{aligned}
$$

The main results can be stated as follows:

Theorem 2. Let $w(t)$ be a solution of (1.1) with Cauchy data $\Phi=\left(\begin{array}{l}\phi \\ \phi\end{array}\right) \in$ $H^{2} \cap H_{1}^{s+1} \times H^{1} \cap H_{1}^{s} \cap L_{2 p}$, where $s>n / 2$. Suppose that $p>1+2 / n(n \geqq 1)$ and $\|\Phi\|_{e}$ $\neq 0$. Then there exists a $\delta>0$ such that if $\|\phi\|_{s+1,1}+\|\psi\|_{s, 1} \leqq \delta$, then $\|W(t)\|_{e}$ does not converge to 0 as $t \rightarrow \infty$.

Theorem 3. Let $w(t)$ be a solution of (1.1) with Cauchy data $\Phi=\left(\frac{\phi}{\phi}\right) \in$ $H^{2} \times H^{1} \cap L_{2 p}$.

(i) Suppose that $p>1+2 / n(n \geqq 1)$. Then there exists $\Phi^{+}=\left(\begin{array}{c}\phi^{+} \\ \phi^{+}\end{array}\right) \in H^{2} \times H^{1}$ such that

$$
U(-t) W(t)-\Phi^{+} \longrightarrow 0 \text { weakly in } H^{2} \times H^{1} \text { as } t \rightarrow \infty .
$$

(ii) Suppose that $1+4 / n<p<\infty$ if $1 \leqq n \leqq 6$ and $1+4 / n<p<n /(n-6)$ if $n \geqq 7$. Then the above $\Phi^{+}$satisfies

$$
\left\|W(t)-U(t) \Phi^{+}\right\|_{e} \rightarrow 0 \quad \text { as } t \rightarrow \infty .
$$

The theory of monotone operators provides the existence of a global solution. Uniqueness, energy equality and inequalites are obtained by standard methods. So we may give a sketch of proof of Theorem 1. The energy decay properties of the linear wave equations with a dissipative term are investigated by Mochizuki $[7,8]$ and Matsumura [6]. For the proof of Theorem 2 we use the same energy method used in Mochizuki [7,8]. In order to prove Theorem 3 , the Strichartz estimate (See Proposition 4.1.) and the energy inequality (1.8) play an important role. 


\section{Proof of Theorem 1}

Since $f^{\prime}(u)>0$ and $f(u) u \geqq 0$, the theory of monotone operators provides a unique solution of (1.1). Noting that $\Phi=\left(\begin{array}{c}\phi \\ \phi\end{array}\right) \in H^{2} \times H^{1} \cap L_{2 p}$ implies $\|\Phi\|_{e}^{2},\|H \phi\|_{e}^{2}$ and $\left\|\left(\begin{array}{c}\phi \\ -H^{2} \phi-f(\psi)\end{array}\right)\right\|_{e}$ are finite, (1.7), (1.8) and (1.9) are obtained by standard methods. So there exist a solution $w(t)$ of (1.1) as follows:

$$
\begin{gathered}
w(t) \in L_{\infty}\left(\mathbb{R}^{+} ; H^{2}\right) \cap C_{w}\left(\boldsymbol{R}^{+} ; H^{2}\right) \cap C_{L}\left(\boldsymbol{R}^{+} ; H^{1}\right), \\
\partial_{t} w(t) \in L_{\infty}\left(\boldsymbol{R}^{+} ; H^{1}\right) \cap C_{w}\left(\boldsymbol{R}^{+} ; H^{1}\right) \cap C_{L}\left(\boldsymbol{R}^{+} ; L_{2}\right) \cap L_{p+1}\left(\boldsymbol{R}^{+} \times \mathbb{R}^{n}\right), \\
\partial_{t}^{2} w(t) \in L_{\infty}\left(\boldsymbol{R}^{+} ; L_{2}\right) \cap C_{w}\left(\boldsymbol{R}^{+} ; L_{2}\right) .
\end{gathered}
$$

Since $\left\|f\left(\partial_{t} w(t)\right)\right\|_{2}=\left\|\partial_{t}^{2} w(t)-\Delta w(t)+w(t)\right\|_{2}$, we have $\partial_{t} w(t) \in L_{\infty}\left(\mathbb{R}^{+} ; L_{2 p}\right)$ by $(2.1)$ and (2.3).

Employing the same arguments as in Kato [4], Shibata [10] and Shibata and Kikuchi [11], we can obtain

$$
w(t) \in C\left(\mathbb{R}^{+} ; H^{2}\right) \cap C^{1}\left(\mathbb{R}^{+} ; H^{1}\right) \cap C^{2}\left(\boldsymbol{R}^{+} ; L_{2}\right) .
$$

Thus Theorem 1 is proved.

\section{Proof of Theorem 2}

We note that $W(t)=\left(\begin{array}{c}w(t) \\ \partial_{t} w(t)\end{array}\right)$ satisfies

$$
W(t)=U(t) \Phi-\int_{0}^{t} U(t-\tau) F\left(\partial_{t} w(\tau)\right) d \tau
$$

where $F(u)=\left(\begin{array}{c}0 \\ f(u)\end{array}\right)$. Since $U(t)$ is an unitary operator on $H^{1} \times L_{2}$, we have

$$
\begin{aligned}
\langle W(t), U(t) \Phi\rangle_{e} & =\langle U(t) \Phi, U(t) \Phi\rangle_{e}-\int_{0}^{t}\left\langle U(t-\tau) F\left(\partial_{t} w(\tau)\right), U(t) \Phi\right\rangle_{e} d \tau \\
& =\|\Phi\|_{e}^{2}-\int_{0}^{t}\left\langle F\left(\partial_{t} w(\tau)\right), U(\tau) \Phi\right\rangle_{e} d \tau \\
& =\|\Phi\|_{e}^{2}-\frac{1}{2} \int_{0}^{t}\left\langle f\left(\partial_{t} w(\tau)\right), \partial_{t} w^{0}(\tau)\right\rangle d \tau
\end{aligned}
$$

Here $w^{0}(t)$ is a solution of the linear Klein-Gordon equation;

$$
\left\{\begin{array}{l}
\partial_{t}^{2} w^{0}(t)-\Delta w^{0}(t)+w^{0}(t)=0 \\
w^{0}(0)=\phi, \quad \partial_{t} w^{0}(0)=\phi
\end{array}\right.
$$

By the Schwarz inequality we obtain 


$$
\begin{aligned}
\|\Phi\|_{e}^{2} & \leqq W(t)\left\|_{e}\right\| U(t) \Phi \|_{e}+\frac{1}{2} \int_{0}^{t}\left|\left\langle f\left(\partial_{t} w(\tau)\right), \partial_{t} w^{0}(\tau)\right\rangle\right| d \tau \\
& =\|W(t)\|_{e}\|\Phi\|_{e}+\frac{1}{2} \int_{0}^{t}\left|\left\langle f\left(\partial_{t} w(\tau)\right), \partial_{t} w^{0}(\tau)\right\rangle\right| d \tau \\
& =I_{1}+I_{2} .
\end{aligned}
$$

It follows from Hölder's inequality that

$$
I_{2} \leqq \frac{1}{2}\left\{\int_{0}^{t} \int_{R^{n}}\left|\partial_{t} w(\tau)\right|^{p+1} d x d \tau\right\}^{p /(p+1)}\left\{\int_{0}^{t} \int_{R^{n}}\left|\partial_{t} w^{0}(\tau)\right|^{p+1} d x d \tau\right\}^{1 /(p+1)}
$$

We recall the well-known estimate

$$
\left\|w^{0}(t)\right\|_{\infty} \leqq C(1+t)^{-n / 2}\left(\|\phi\|_{s, 1}+\|\phi\|_{s-1,1}\right),
$$

where $s>n / 2$ and $w^{0}(t)$ is a solution of (3.3). (See Brenner [2] Appendix 2, Bergh and Löfström [1] Theorem 6.2.4 and Brenner, Thomée and Wablbin [3] Theorem 2.1.) By (1.7) and $\left\|\partial_{t} w^{0}(t)\right\|_{2}^{2} \leq 2\|\Phi\|_{e}^{2}$ we have

$$
\begin{aligned}
& I_{2} \leqq C\left(\|\phi\|_{s+1,1}+\|\phi\|_{s, 1}\right)^{(p-1) /(p+1)}\|\Phi\|_{e}^{2 p /(p+1)} \\
& \quad \times\left\{\int_{0}^{t}(1+\tau)^{-n(p-1) / 2}\left\|\partial_{t} w^{0}(\tau)\right\|_{2}^{2} d \tau\right\}^{1 /(p+1)} \\
& \leqq C\left(\|\phi\|_{s+1,1}+\|\phi\|_{s, 1}\right)^{(p-1) /(p+1)}\|\Phi\|_{e}^{2}\left\{\int_{0}^{t}(1+\tau)^{-n(p-1) / 2} d \tau\right\}^{1 /(p+1)} .
\end{aligned}
$$

Since $p>1+2 / n$ implies $-n(p-1) / 2<-1$, there exists a $\delta>0$ such that

$$
C \delta^{(p-1) /(p+1)}\left\{\int_{0}^{\infty}(1+\tau)^{-n(p-1) / 2} d \tau\right\}^{1 /(p+1)}<\frac{1}{2} .
$$

Then it follows from (3.4), (3.7) and (3.8) that

$$
\|\phi\|_{e}^{2} \leqq\|W(t)\|_{e}\|\Phi\|_{e}+\frac{1}{2}\|\Phi\|_{e}^{2}
$$

if $\|\dot{\phi}\|_{s+1,1}+\|\phi\|_{s, 1} \leqq \delta$. Noting that $\|\Phi\|_{e} \neq 0$, we have $\frac{1}{2}\|\Phi\|_{e}^{2} \leqq\|W(t)\|_{e}$ for any $t \in \mathbb{R}^{+}$. Therefore Theorem 2 is proved.

\section{Proof of Theorem 3}

We begin with the Strichartz estimate for solutions of the linear KleinGordon equation.

Proposition 4.1. Let $q \geqq 2, r \geqq 2$ and

$$
\frac{1}{2}-\frac{1}{n}-\frac{1}{n r}<\frac{1}{q}<\frac{1}{2}-\frac{2}{n r} \text {. }
$$


Then we have

$$
\left\|w^{0}\right\|_{L_{r}\left(R ; L_{q}\left(R^{n}\right)\right)} \leqq C\|\Phi\|_{e},
$$

where $\Phi=\left(\begin{array}{l}\phi \\ \phi\end{array}\right)$ and $w^{0}(t)$ is a solution of (3.3).

See Marshall [5] for a proof.

Using this proposition, we obtain the following

Lemma 4.2. Under the same assumptions of Proposition 4.1 we have

$$
\begin{aligned}
\left\|\int_{t}^{\infty} U(-\tau) F(u(\tau)) d \tau\right\|_{e} \leq & C\left\||u|^{(p-1) / 1}(|\nabla u|+|u|)\right\|_{L_{2}\left([t, \infty) \times R^{n}\right)} \\
& \times\|u\|_{L_{r(p-1) /(r-2)}^{(p-1) / 2}}^{\left([t, \infty) ; L_{q(p-1) /(q-2)}\left(R^{n}\right)\right)}
\end{aligned}
$$

for suitable functions $u$, where $F(u)=\left(\begin{array}{c}0 \\ f(u)\end{array}\right)$.

Proof. For any $V=\left(\begin{array}{l}v_{1} \\ v_{2}\end{array}\right) \in C_{0}^{\infty}\left(\boldsymbol{R}^{n}\right) \times C_{0}^{\infty}\left(\boldsymbol{R}^{n}\right)$ we have

$$
\begin{aligned}
\left\langle\int_{t}^{\infty} U(-\tau) F(u(\tau)) d \tau, V\right\rangle_{e} & =\int_{t}^{\infty}\langle U(-\tau) F(u(\tau)) d \tau, V\rangle_{e} d \tau \\
& =\int_{t}^{\infty}\langle F(u(\tau)), U(\tau) V\rangle_{e} d \tau \\
& =\frac{1}{2} \int_{t}^{\infty}\left\langle H f(u(\tau)), H^{-1} \partial_{t} v(\tau)\right\rangle d \tau,
\end{aligned}
$$

where $v(t)=\cos \{H t\} v_{1}+H^{-1} \sin \{H t\} v_{2}$. Recalling that $H=\sqrt{-\Delta+1}$, by Hölder's inequality we have

$$
\begin{aligned}
& \left|\int_{\tau}^{\infty}\left\langle H f(u(\tau)), H^{-1} \partial_{t} v(\tau)\right\rangle d \tau\right| \\
& \leqq C \int_{t}^{\infty} \int_{R^{n}}|u|^{(p-1) / 2}(|\nabla u|+|u|)|u|^{(p-1) / 2}\left|H^{-1} \partial_{t} v(\tau)\right| d x d \tau \\
& \leqq C\left\||u|^{(p-1) / 2}(|\nabla u|+|u|)\right\|_{L_{2}\left([t, \infty) \times R^{n}\right)} \\
& \times\|u\|_{L_{r(p-1) /(r-2)}^{(p-1) / 2}\left([t, \infty) ; L_{q(p-1) /(q-2)}\right.}\left\|H^{-1} \partial_{t} v\right\|_{L_{r}\left([t, \infty) ; L_{q}\left(R^{n}\right)\right)},
\end{aligned}
$$

where $q \geqq 2$ and $r \geqq 2$. Since $H^{-1} \partial_{t} v(t)$ is a solution of (3.3) with Cauchy data $\left(\begin{array}{c}H^{-1} v_{2} \\ -H v_{1}\end{array}\right)$, it follows from Proposition 4.1, (4.4) and (4.5) that 


$$
\begin{aligned}
& \left|\left\langle\int_{t}^{\infty} U(-\tau) F(u(\tau)) d \tau, V\right\rangle_{e}\right| \\
& \leqq \\
& \quad C\left\||u|^{(p-1) / 2}(|\nabla u|+|u|)\right\|_{L_{2}\left([t, \infty) \times R^{n}\right)} \\
& \quad \times\|u\|_{L_{r(p-1) /(r-2)}^{(p-1) / 2}\left([t, \infty) ; L_{q(p-1) /(q-2)}\left(R^{n}\right)\right)}^{(p)}\|V\|_{e} .
\end{aligned}
$$

Thus by the duality argument we obtain (4.3).

Q.E.D.

Proof of (i). We note that $W(t)$ satisfies

$$
W(t)=U(t) \Phi-\int_{0}^{t} U(t-\tau) F\left(\partial_{t} w(\tau)\right) d \tau,
$$

and then

$$
\begin{aligned}
U(-t) W(t) & =\Phi-\int_{0}^{t} U(-\tau) F\left(\partial_{t} w(\tau)\right) d \tau \\
& =\Phi^{+}+\int_{t}^{\infty} U(-\tau) F\left(\partial_{t} w(\tau)\right) d \tau
\end{aligned}
$$

where

$$
\Phi^{+}=\Phi-\int_{0}^{\infty} U(-\tau) F\left(\partial_{t} w(\tau)\right) d \tau
$$

For $V=\left(\begin{array}{c}v_{1} \\ v_{2}\end{array}\right) \in C_{0}^{\infty}\left(\boldsymbol{R}^{n}\right) \times C_{0}^{\infty}\left(\boldsymbol{R}^{n}\right)$ we have

$$
\begin{aligned}
\left\langle U(-t) W(t)-\Phi^{+}, V\right\rangle_{e} & =\int_{0}^{\infty}\left\langle U(-\tau) F\left(\partial_{t} w(\tau)\right), V\right\rangle_{e} d \tau \\
& =\int_{t}^{\infty}\left\langle F\left(\partial_{t} w(\tau)\right), U(\tau) V\right\rangle_{e} d \tau \\
& =\frac{1}{2} \int_{t}^{\infty}\left\langle f\left(\partial_{t} w(\tau)\right), \partial_{t} v(\tau)\right\rangle d \tau,
\end{aligned}
$$

where $v(t)=\cos \{H t\} v_{1}+H^{-1} \sin \{H t\} v_{2}$. In the same way as in obtaining (3.5) and (3.7), it holds that for $p>1+2 / n$

$$
\left|\left\langle U(-t) W(t)-\phi^{+}, V\right\rangle_{e}\right| \longrightarrow 0 \quad \text { as } t \rightarrow \infty \text {. }
$$

Since $U(t)$ is an unitary operator on $H^{1} \times L_{2}$, it follows from (1.8) that $\{U(-t) W(t)\}$ is uniformly bounded on $t$ in $H^{2} \times H^{1}$. Therefore we have

$$
U(-t) W(t) \longrightarrow \Phi^{+} \quad \text { weakly in } H^{2} \times H^{1} \text { as } t \rightarrow \infty
$$

and $\Phi^{+} \in H^{2} \times H^{1}$.

Proof of (ii). If $1 / r$ and $1 / q$ satisfy (4.1), by (4.8) and Lemma 4.2 we have 


$$
\begin{aligned}
\left\|W(t)-U(t) \Phi^{+}\right\|_{e}= & \left\|U(-t) W(t)-\Phi^{+}\right\|_{e} \\
\leqq & \left\|\int_{t}^{\infty} U(-\tau) F\left(\partial_{t} w(\tau)\right) d \tau\right\|_{e} \\
\leqq & C\left\|\left|\partial_{t} w\right|^{(p-1) / 2}\left(\left|\nabla \partial_{t} w\right|+\left|\partial_{t} w\right|\right)\right\|_{L_{2}\left([t, \infty) \times R^{n}\right)} \\
& \times\left\|\partial_{t} w\right\|_{L_{r(p-1) /(r-2)}^{(p-1) / 2}\left([t, \infty) ; L_{q(p-1) /(q-2)}\left(R^{n}\right)\right)} .
\end{aligned}
$$

On the other hand by (1.8) and (1.5) we have

$$
\left|\partial_{t} w\right|^{(p-1) / 2}\left(\left|\nabla \partial_{t} w\right|+\left|\partial_{t} w\right|\right) \in L_{2}\left(\boldsymbol{R}^{+} \times \mathbb{R}^{n}\right)
$$

and $\partial_{t} w \in L_{r^{\prime}}\left(\boldsymbol{R}^{+} ; L_{q^{\prime}}\left(\mathbb{R}^{n}\right)\right)$, where $1 / r^{\prime}=\theta / p+1$ and $1 / q^{\prime}=(\theta / p+1)+(1-\theta) / 2 p$ $(0 \leqq \theta \leqq 1)$. Thus if

$$
\frac{r-2}{r(p-1)}=\frac{1}{r^{\prime}}, \quad \frac{q-2}{q(p-1)}=\frac{1}{q^{\prime}},
$$

it follows from (4.13) that

$$
\left\|W(t)-U(t) \phi^{+}\right\|_{e} \longrightarrow 0 \quad \text { as } t \rightarrow \infty
$$

(4.14) implies that

$$
\frac{1}{r}=\frac{1}{2}-\frac{(p-1) \theta}{2(p+1)}, \quad \frac{1}{q}=\frac{1}{2}-\frac{(p-1) \theta}{2(p+1)}-\frac{(p-1)(1-\theta)}{4 p} .
$$

Substituting (4.16) for (4.1), we have

$$
\begin{aligned}
& \{(n+2) \theta+(n-6)\} p^{2}-2\{(n+1) \theta+3\} p-n(1-\theta)<0, \\
& \{(n+4) \theta+(n-4)\} p^{2}-2\{(n+2) \theta+2\} p-n(1-\theta)>0 .
\end{aligned}
$$

By (4.17) we have

$$
\begin{gathered}
1<p<\infty \quad \text { if } \theta \leqq \frac{6-n}{n+2}, \\
1<p<\alpha_{n}(\theta) \quad \text { if } \theta>\frac{6-n}{n+2} .
\end{gathered}
$$

Here $\alpha_{n}(\theta)$ is a positive solution of

$$
\{(n+2) \theta+(n-6)\} p^{2}-2\{(n+1) \theta+3\} p-n(1-\theta)=0 .
$$

On the other hand by (4.18) we have

$$
p>\beta_{n}(\theta) \text { if } \theta>\frac{4-n}{n+4} .
$$

Here $\beta_{n}(\theta)$ is a positive solution of

$$
\{(n+4) \theta+(n-4)\} p^{2}-2\{(n+2) \theta+2) p-n(1-\theta)=0 .
$$


Noting that

$$
\begin{aligned}
\{(n+2) \theta+(n-6)\} p^{2}-2\{(n+1) \theta+3\} p-n(1-\theta) \\
<\{(n+4) \theta+(n-4)\} p^{2}-2\{(n+2) \theta+2\} p-n(1-\theta)
\end{aligned}
$$

for $p>1$, we see that $\beta_{n}(\theta)<\alpha_{n}(\theta)$ for $(6-n) /(n+2)<\theta$.

First we consider the case $1 \leqq n \leqq 6$. Since $\alpha_{n}(\theta) \uparrow \infty$ as $\theta \downarrow(6-n) /(n+2)$, there exists an $\varepsilon>0$ such that

$$
\beta_{n}\left(\frac{6-n}{n+2}\right)<\alpha_{n}\left(\frac{6-n}{n+2}+\varepsilon\right) .
$$

Since $0 \leqq \theta \leqq 1$, it follows from (4.19), (4.20) and (4.21) that

$$
\begin{gathered}
\beta_{n}(\theta)<p<\infty \quad \text { for } \operatorname{Max}\left\{0, \frac{4-n}{n+4}\right\}<\theta \leqq \frac{6-n}{n+2}, \\
\beta_{n}(\theta)<p<\alpha_{n}(\theta) \quad \text { for } \frac{6-n}{n+2}<\theta \leqq 1
\end{gathered}
$$

respectively. Noting that $\alpha_{n}(\theta)$ and $\beta_{n}(\theta)$ are monotone decreasing functions, we have

$$
\begin{gathered}
\beta_{n}\left(\frac{6-n}{n+2}\right)<p<\infty, \\
\beta_{n}(1)<p<\alpha_{n}\left(\frac{6-n}{n+2}+\varepsilon\right) .
\end{gathered}
$$

Thus by (4.22), (4.25) and (4.26) we have $1+4 / n=\beta_{n}(1)<p<\infty$ if $1 \leqq n \leqq 6$.

Next we consider the case $n \geqq 7$. By (4.20) and (4.21) we have

$$
\beta_{n}(\theta)<p<\alpha_{n}(\theta) \quad \text { for } 0 \leqq \theta \leqq 1 .
$$

Since $\alpha_{n}(\theta)$ and $\beta_{n}(\theta)$ are monotone decreasing functions, we have

$$
1+\frac{4}{n}=\beta_{n}(1)<p<\alpha_{n}(0)=\frac{n}{n-6} .
$$

Thus Theorem 3 is proved.

\section{References}

[1] Bergh, J. and Löfström, J., Interpolation Spaces, Berlin-Heidelberg-New York, Springer-Verlag, 1976.

[2] Brenner, P., On scattering and everywhere defined scattering operator for nonlinear Klein-Gordon equations, J. Differential Equations, 56 (1985), 310-344.

[3] Brenner, P. and Thomée, V. and Wahlbin, L. B., Besov Spaces and Applications to Difference Methods for Initial Value Problems, Lecture notes in mathematics, 434 (1975), Berlin-Heidelberg-New York, Springer-Verlag. 
[4] Kato, T., Abstract differential equations and nonlinear mixed problem, Scuola Normale Superiore, Lezioni Fermiane, Pisa (1985).

[5] Marshall, B., Mixed norm estimates for the Klein-Gordon equation, Proceedings of a Conference on Harmonic Analysis in Honor of A, Zygmund 1981 vol. 2, 638-645, Springer-Verlag, 1983.

[6] Matsumura, A., Energy decay of solutions of dissipative wave equations, Proc. Japan Acad., 53, Ser. A (1977), 232-237.

[7] Mochizuki, K., Scattering theory for wave equations with dissipative terms, $P u b l$. RIMS, Kyoto Univ., 12 (1976), 383-390.

[8] - The scattering theory for wave equations, Kinokuniya, 1984. [Japanese]

[9] Nakao, M., Energy decay of the wave equation with a nonlinear dissipative term, Funkcialaj Ekvacioj, 26 (1983), 237-250.

[10] Shibata, Y., On the Neumann problem for some linear hyperbolic systems of 2nd order with coefficients in Sobolev spaces, Tsukuba J. Math., 13 (1988), 283352.

[11] Shibata, Y. and Kikuchi, M., On the mixed problem for some quasilinear hyperbolic system with fully nonlinear boundary condition, J. Differential Equations, 80 (1986), 154-197.

[12] Strauss, W.A., The energy method in nolinear partial differential equations, Brasil Inst. Mat. Pura e Aplicada, 1969.

\author{
Japanese Language School \\ The Tokyo University of \\ Foreign Studies \\ Fucyu, Tokyo, 183 Japan
}

